\title{
NOS-2 participates in the behavioral effects of ethanol withdrawal in zebrafish
}

Suianny Nayara da Silva Chaves ${ }^{1}$, Bruna Patricia Dutra Costa ${ }^{1,2}$, Gabriela Cristini Vidal Gomes ${ }^{1}$, Monica Lima-Maximino ${ }^{3}$, Eduardo Pacheco Rico $^{4}$, Caio Maximino ${ }^{1 *}$

${ }^{1}$ Laboratório de Neurociências e Comportamento “Frederico Guilherme Graeff”, Faculdade de Psicologia, Universidade Federal do Sul e Sudeste do Pará, Marabá/PA, Brazil

${ }^{2}$ Rede de Biodiversidade e Biotecnologia da Amazônia Legal, Pólo Marabá, Marabá/PA, Brazil

${ }^{3}$ Laboratório de Neurofarmacologia e Biofísica, Centro de Ciências Biológicas e da Saúde,

Departamento de Morfologia e Ciências Fisiológicas, Universidade do Estado do Pará, Campus

VIII, Marabá/PA, Brazil

${ }^{4}$ Translational Psychiatry Laboratory, Graduate Program in Health Sciences, University of Southern Santa Catarina (UNESC), Criciúma/SC, Brazil.

* Corresponding author

Caio Maximino

Laboratório de Neurociências e Comportamento - Instituto de Estudos em Saúde e Biológicas, Universidade Federal do Sul e Sudeste do Pará, Unidade III

Av. dos Ipês, S/N, s/ CEP, Bairro Cidade Jardim, Marabá/PA, Brazil

cmaximino@unifesspa.edu.br 


\begin{abstract}
Nitric oxide has been implicated in symptoms of ethanol withdrawal in animal models. Zebrafish have been used as models to study neurobehavioral effects of ethanol $(\mathrm{EtOH})$ withdrawal, but the mechanisms associated with these effects are not yet clear. Adult zebrafish were treated with $1 \%$ EtOH for 20 min per day for 8 days, injected with the nitric oxide synthase 2 (NOS-2) inhibitor aminoguanidine $(50 \mathrm{mg} / \mathrm{kg})$, and allowed to experience withdrawal (WD) in their hometanks for 7 days. EtOH WD increased anxiety-like behavior in the novel tank test, an effect that was blocked by aminoguanidine. EtOH WD also increased brain levels of nitrite, an effect that was partially blocked by aminoguanidine. These results underline a novel mechanism by which NOS-2 controls anxiety-like responses to ethanol withdrawal, with implications for the mechanistic study of symptoms associated with chronic ethanol abuse.
\end{abstract}

Preprint: https://dx.doi.org/10.20944/preprints201912.0219.v1

Data and scripts: https://github.com/lanec-unifesspa/etoh-withdrawal/tree/master/NOS2 Keywords: Nitric oxide; Oxidative stress; Ethanol withdrawal; Anxiety

\title{
1. Introduction
}

Nitric oxide synthase 2 (NOS-2), an inducible isozyme which participates in the biosynthesis of nitric oxide under conditions of stress and neuroinflammation [1], has been shown to be involved in the formation of peroxynitrite and other nitrogen reactive species [2]. Due to the fact that this isozyme is not constitutionally expressed and has calcium-independent activity, it has been implicated in stress sensibilization mechanisms: in situations of sustained physiological stress, the expression of NOS-2 is induced, and continually produces nitric oxide as long as there is available subtrate and the enzyme is not degraded. Nitric oxide has been implicated in the mechanisms of withdrawal syndrome and alcoholic abstinence syndrome, characteristic dysphoric states that follows the abrupt cessation of drug and/or alcohol use; in preclinical research, inhibiting nitric 
oxide synthesis diminishes withdrawal-like symptoms in rats treated with ethanol [3], and ethanol withdrawal (EtOH-WD) activates nitric oxide-producing neurons in anxiety-related areas [4]. We have previously confirmed, through meta-analysis and a conceptual replication, that EtOH-WD increases anxiety-like behavior in adult zebrafish, an effect that is accompanied by decreased activity of the antioxidant enzyme catalase in the brain [5]. Moreover, EtOH-WD has been shown to induce oxidative imbalance in the zebrafish brain, and the antioxidant $N$-acetylcysteine blocks both effects [6]. These results suggested that oxidative and nitrosative stress can be involved in the pathophysiology of EtOH-WD. In zebrafish, NOS-2 is coded by two different genes, nos $2 a$ and $n o s 2 b$, which are expressed in the central nervous system and upregulated after inflammatory stimuli [7], suggesting that these are also inducible isoforms. In the present work, we test the hypothesis that EtOH-WD-elicited anxiety is also mediated by NOS-2 in zebrafish. This manuscript is a complete report of all the studies performed to test the effect of aminoguanidine on anxiety-like behavior in zebrafish after EtOH-WD.

\section{Materials and methods}

42 adult ( 4 mo., standard length 23.1 - $34.8 \mathrm{~mm}$ ) zebrafish from the longfin phenotype were acquired in a local aquarium shop and kept in collective tanks (40 L, 5 animals/L) for at least two weeks before experiments begun, and fed twice daily on commercial dry feed (Alcon® Gold Fish Colour). Animals used in the experiments were of mixed sexes, and kept in an approximate proportion of 50 females:50 males, based on body morphology. Animals were randomly drawn from the tank and allocated to experimental conditions (below); randomization was achieved by using a random number generator (http://www.jerrydallal.com/random/random_block_size_r.htm), with each subject randomized to a single treatment using random permuted blocks. One PI attributed a random letter to treatment (e.g., "A" for control, "B" for withdrawal) and a random integer for drug dose (e.g., "1" for $50 \mathrm{mg} / \mathrm{kg}$, "2" for $0 \mathrm{mg} / \mathrm{kg}$ [vehicle]), and combinations for 
letters and integers were randomized. For each experiment, animals were treated and tested in the order of allocation (i.e., randomly). In all experiments, experimenters and data analysts were blinded to drugs and treatment by using coded vials (with the same code used for randomization); blinding was removed only after data analysis. Experiments were always run between 08:00AM and 02:00 PM. Sample sizes were defined based on the standard mean difference derived from a metanalysis of the effects EtOH-WD on anxiety-like behavior in zebrafish, considering $80 \%$ power to detect a similar effect [5], and were calculated based on a two-way ANOVA model using the R package 'pwr2' (v. 1.0) [8]. Water conditions, housing, and feeding conditions were standardized as per recommendations for zebrafish $[9,10]$. Water quality parameters can be found online (https://github.com/lanec-unifesspa/lanec-welfare); experiments were made between October and December 2018. Potential suffering of animals was minimized by controlling for the aforementioned environmental variables and scoring humane endpoints (clinical signs, behavioral changes, bacteriological status), following Brazilian legislation [9]. Experiments were approved by UEPA's IACUC under protocol 06/18.

Animals were exposed to a regimen based on Mathur and Guo [11], with exposure to either water or $1 \% \mathrm{EtOH}$ in $2 \mathrm{~L}$ containers for 20 min per day (exposure at 10:00 AM) for 8 days. EtOH was mixed in the water just before putting the fish in, and animals were exposed in groups of 5-6 animals. Immediately after the end of the exposure period (i.e., on day 8), animals were anesthetized in icecold water (11-12 $\left.{ }^{\circ} \mathrm{C}\right)[12]$, and injected intraperitoneally with either vehicle (Cortland's salt solution) or $50 \mathrm{mg} / \mathrm{kg}$ of the NOS-2 inhibitor aminoguanidine (AG). The injection was made based on standard protocols [13] using a $10 \mu \mathrm{L}$ microsyringe (Hamilton ${ }^{\circledR} 701 \mathrm{~N}$ syringe $701 \mathrm{~N}$, volume 10 $\mu \mathrm{L}$, needle size 26 s gauge at the cone tip), in a final volume of $5 \mu \mathrm{L}$. The dose was based on experiments made in rodents that showed that this dose blocks the anxiogenic effects of restraint stress [14] and the sensitizing effects of stress-restress [15]. After this period, animals were allowed to experience withdrawal from $\mathrm{EtOH}$ in their hometanks for 7 days. After that, animals were tested 
in the novel tank test [16], using a 5 L rectangular tank (A cm X $24 \mathrm{~cm} \mathrm{X} 22 \mathrm{~cm}$, w X 1 X h) that was freely explored for 6 min; behavioral variables were defined as per Lima et al. [17]. Light levels above the tanks were measured using a handheld light meter, and ranged from 251 to 280 lumens (coefficient of variation $=3.399 \%$ between subjects).

Animals were then sacrificed in ice-cold water [12], decapitated, and their brains were dissected, forming pools of 3 brains. Nitrite content in these matrices were analyzed using a modified Griess protocol [18], and reported as micromoles per milligram of protein. Data were analyzed using robust ANOVAs with permutation tests [19], with p-values adjusted for the false discovery rate using the Benjamini-Hochberg procedure [20]. Alpha was set at 0.05. Analyses were made using the package 'rcompanion' for R [21]. Data and analysis scripts were posted on GitHub (https://github.com/lanec-unifesspa/etoh-withdrawal/tree/master/NOS2). We report how the sample size was determined, all data exclusions (if any), all manipulations, and all measures in the study.

\section{Results}

Main effects of withdrawal status $(\mathrm{p}=0.0304)$ and drug $(\mathrm{p}=0.0458)$, as well as an interaction effect $(p=0.0276)$, were found for time on bottom (Fig. 1A, 1A'). No main effects of withdrawal status $(\mathrm{p}=0.172)$ or drug $(\mathrm{p}=0.1488)$ were found for time on top, but an interaction effect was found $(p=0.001$; Fig. 1B, 1B'). No main effects of withdrawal status $(p=0.1454)$ or drug $(p=$ 0.0738), nor interaction effects $(\mathrm{p}=0.1610)$, were found for erratic swimming (Fig. 2A). No main effects of withdrawal status $(p=0.3338)$ or drug $(p=0.583)$, nor interaction effects $(p=0.764)$, were found for thrasing (Fig. 2B). No main effects of withdrawal status $(p=0.5028)$ or drug $(p=$ 0.0902), nor interaction effects $(\mathrm{p}=0.1102)$, were found for freezing frequency (Fig. 2C). No main effects of withdrawal status $(\mathrm{p}=0.0834)$ nor a drug effect $(\mathrm{p}=0.918)$ were found for freezing duration (Fig. 2D), but an interaction effect was found ( $\mathrm{p}=0.0446)$. No main effects of withdrawal status $(p=0.244)$ or drug $(p=0.3268)$, nor an interaction effect $(p=0.6128)$, were found for total 
locomotion (Fig. 2E). Main effects of withdrawal status $\left(p=6.45 \times 10^{-5}\right)$, drug $(p=0.00182)$, and interaction $(\mathrm{p}=0.00757)$ were found for nitrite levels (Fig. 2F).

\section{Discussion}

The increases in geotaxis and freezing after withdrawal is consistent with previous findings in EtOH WD in zebrafish [5], and are indicative of increased anxiety, stress, or arousal [22]. WD also increased nitrite levels in the brain, an effect that was blocked by treatment with aminoguanidine. Aminoguanidine, a NOS-2 inhibitor, also prevented the effects of WD on anxietylike behavior in the NTT, suggesting that both the increases in nitrite and the anxiogenic-like effect of ethanol withdrawal are associated with this enzyme. Since EtOH-WD also produces oxidative imbalances in the zebrafish brain [5,6], it is possible that the nitrite production observed here represents nitrosative stress. Chronic ethanol exposure also produces oxidative stress, but not increased nitrite, in the zebrafish brain [23], suggesting that the effect on the nitrergic pathway is specific to withdrawal. Both oxidative stress [24,25] and nitric oxide [26,27] have been implicated in stress-induced anxiogenesis in zebrafish, suggesting that the nitrergic system is a common mediator of the behavioral effects of stressors. EtOH-WD has also been shown, in rodents, to sensitize neurons associated with defensive behavior $[28,29]$. Thus, the present results suggest that EtOH-WD increases nitric oxide production in the brain through NOS-2, and that this increased NO leads to increased anxiety. Further work is needed to understand how this NOS-2-mediated mechanism interacts with other known mechanisms of withdrawal-sensitized behavior.

\section{Acknowledgments}

SNSC was the recipient of a Fundação Amazônia de Amparo a Estudos e Pesquisas (FAPESPA) scholarship. 


\section{References}

[1] D.S. Bredt, Endogenous nitric oxide synthesis: Biological functions and pathophysiology, Free Radic. Res. 31 (1999) 577-596. https://doi.org/10.1080/10715769900301161.

[2] D.D. Thomas, J.L. Heinecke, L.A. Ridnour, R.Y. Cheng, A.H. Kesarwala, C.H. Switzer, D.W. McVicar, D.D. Roberts, S. Glynn, J.M. Fukuto, D.A. Wink, K.M. Miranda, Signaling and stress: The redox landscape in NOS2 biology, Free Radic. Biol. Med. 87 (2015) 204-225. https://doi.org/10.1016/j.freeradbiomed.2015.06.002.

[3] I.T. Uzbay, B.F. Erden, E.E. Tapanyigit, S.O. Kayaalp, Nitric oxide synthase inhibition attenuates signs of ethanol withdrawal in rats, Life Sci. 61 (1997) 2197-2209. https://doi.org/10.1016/S0024-3205(97)00922-3.

[4] V.T. Bonassoli, H. Milani, R.M.W. de Oliveira, Ethanol withdrawal activates nitric oxideproducing neurons in anxiety-related brain areas, Alcohol. 45 (2011) 641-652. https://doi.org/10.1016/j.alcohol.2010.11.007.

[5] S.N.S. Chaves, G.R. Felício, B.P.D. Costa, W.E.A. de Oliveira, M.G. Lima-Maximino, D.H. de Siqueira-Silva, C. Maximino, Behavioral and biochemical effects of ethanol withdrawal in zebrafish, Pharmacol. Biochem. Behav. 169 (2018) 48-58.

https://doi.org/10.1016/j.pbb.2018.04.006.

[6] R. Mocelin, M. Marcon, A.S. da Rosa Araujo, A.P. Herrmann, A. Piato, Withdrawal effects following repeated ethanol exposure are prevented by N-acetylcysteine in zebrafish, Prog. Neuro-Psychopharmacology Biol. Psychiatry. 93 (2019) 161-170. https://doi.org/10.1016/j.pnpbp.2019.03.014.

[7] S. Lepiller, N. Franche, E. Solary, J. Chluba, V. Laurens, Comparative analysis of zebrafish $n o s 2 a$ and nos $2 b$ genes, Gene. 445 (2009) 58-65. https://doi.org/10.1016/j.gene.2009.05.016.

[8] P. Lu, J. Liu, D. Koestler, pwr2: Power and Sample Size Analysis for One-way and Two-way ANOVA Models, (2017). https://cran.r-project.org/package=pwr2.

[9] Conselho Nacional de Controle de Experimentação Animal - CONCEA, Diretriz brasileira para o cuidado e a utilização de animais para fins científicos e didáticos - DBCA. Anexo I. Peixes mantidos em instalações de instituições de ensino ou pesquisa científica, Brasil, 2017.

[10] C. Lawrence, The husbandry of zebrafish (Danio rerio): A review, Aquaculture. 269 (2007) 1-20. https://doi.org/10.1016/j.aquaculture.2007.04.077.

[11] P. Mathur, S. Guo, Differences of acute versus chronic ethanol exposure on anxiety-like behavioral responses in zebrafish, Behav. Brain Res. 219 (2011) 234-239. https://doi.org/10.1016/j.bbr.2011.01.019.

[12] J.M. Wilson, R.M. Bunte, A.J. Carty, Evaluation of rapid cooling and tricaine methanesulfonate (MS222) as methods of euthanasia in zebrafish (Danio rerio), J. Am. Assoc. Lab. Anim. Sci. 48 (2009) 785-789. 
[13] M.D. Kinkel, S.C. Eames, L.H. Philipson, V.E. Prince, Intraperitoneal injection into adult zebrafish, J. Vis. Exp. 42 (2010) 2126. https://doi.org/10.3791/2126.

[14] N. Gilhotra, D. Dhingra, Involvement of NO-cGMP pathway in anti-anxiety effect of aminoguanidine in stressed mice, Prog. Neuro-Psychopharmacology Biol. Psychiatry. 33 (2009) 1502-1507. https://doi.org/10.1016/j.pnpbp.2009.08.012.

[15] B.H. Harvey, F. Oosthuizen, L. Brand, G. Wegener, D.J. Stein, Stress-restress evokes sustained iNOS activity and altered GABA levels and NMDA receptors in rat hippocampus, Psychopharmacology (Berl). 175 (2004) 494-502. https://doi.org/10.1007/s00213-004-18364.

[16] J.M. Cachat, A.M. Stewart, L. Grossman, S. Gaikwad, F. Kadri, E. Al., K.M. Chung, N. Wu, K. Wong, S. Roy, C. Suciu, J. Goodspeed, M. Elegante, B. Bartels, S. Elkhayat, D. Tien, J. Tan, A. Denmark, T. Gilder, E. Kyzar, J. Dileo, K. Frank, K. Chang, E. Utterback, P. Hart, A. V Kalueff, Measuring behavioral and endocrine responses to novelty stress in adult zebrafish, Nat. Protoc. 5 (2010) 1786-1799. https://doi.org/10.1038/nprot.2010.140.

[17] M.G. Lima, R.X. do C. Silva, S. de N. dos S. Silva, L. do S. dos S. Rodrigues, K.R.H.M. Oliveira, E. de J.O. Batista, C. Maximino, A.M. Herculano, Time-dependent sensitization of stress responses in zebrafish: A putative model for post-traumatic stress disorder, Behav. Processes. 128 (2016) 70-82. https://doi.org/10.1016/j.beproc.2016.04.009.

[18] C. Maximino, J.S. de Oliveira, M.G. Lima-Maximino, Optimized protocol for quantification of nitrite levels in brain and head kidney tissue samples in adult zebrafish, Protocols.Io. (2018). https://doi.org/10.17504/protocols.io.sabeaan.

[19] P.J. Huber, Robust statistics, John Wiley \& Sons, Inc, New York, NY, 1981. https://doi.org/10.1002/0471725250.

[20] Y. Benjamini, D. Drai, G. Elmer, N. Kafkafi, I. Golani, Controlling the false discovery rate in behavior genetics research, Behav. Brain Res. 125 (2001) 279-284. https://doi.org/10.1016/S0166-4328(01)00297-2.

[21] S. Mangiafico, rcompanion: Functions to Support Extension Education Program Evaluation, (2017). https://cran.r-project.org/package=rcompanion.

[22] R.J. Egan, C.L. Bergner, P.C. Hart, J.M. Cachat, P.R. Canavello, M.F. Elegante, S.I. Elkhayat, B.K. Bartels, A.K. Tien, D.H. Tien, S. Mohnot, E. Beeson, E. Glasgow, H. Amri, Z. Zukowska, A. V Kalueff, Understanding behavioral and physiological phenotypes of stress and anxiety in zebrafish, Behav. Brain Res. 205 (2009) 38-44. https://doi.org/10.1016/j.bbr.2009.06.022.

[23] J.F. Agostini, H.C.Z.D. Toé, K.M. Vieira, S.L. Baldin, N.L.F. Costa, C.U. Cruz, L. Longo, M.M. Machado, T.R. da Silveira, P.F. Schuck, E.P. Rico, Cholinergic System and Oxidative Stress Changes in the Brain of a Zebrafish Model Chronically Exposed to Ethanol, Neurotox. Res. 33 (2018) 749-758. https://doi.org/10.1007/s12640-017-9816-8. 
[24] G. Dal Santo, G.M.M. Conterato, L.J.G. Barcellos, D.B. Rosemberg, Â.L. Piato, Acute restraint stress induces an imbalance in the oxidative status of the zebrafish brain, Neurosci. Lett. 558 (2014) 103-108. https://doi.org/10.1016/j.neulet.2013.11.011.

[25] R. Mocelin, A.P. Herrmann, M. Marcon, C.L. Rambo, A. Rohden, F. Bevilaqua, M.S. De Abreu, L. Zanatta, E. Elisabetsky, L.J.G. Barcellos, D.R. Lara, A.L. Piato, N-acetylcysteine prevents stress-induced anxiety behavior in zebrafish, Pharmacol. Biochem. Behav. 139 (2015) 121-126. https://doi.org/10.1016/j.pbb.2015.08.006.

[26] A.M. Herculano, B. Puty, V. Miranda, M.G. Lima, C. Maximino, Interactions between serotonin and glutamate-nitric oxide pathways in zebrafish scototaxis, Pharmacol. Biochem. Behav. 129 (2015) 97-104. https://doi.org/10.1016/j.pbb.2014.12.005.

[27] M.G. Lima, S. de N. dos S. Silva, R.X. do C. Silva, K.R.H.M. Oliveira, E. de J.O. Batista, C. Maximino, A.M. Herculano, Putative involvement of the nitrergic system on the consolidation, but not initiation, of behavioral sensitization after conspecific alarm substance in zebrafish, Pharmacol. Biochem. Behav. 139 (2015) 127-133. https://doi.org/10.1016/j.pbb.2015.08.005.

[28] A. Cabral, N. Isoardi, C. Salum, C.E. de Macedo, M.J. Nobre, V.A. Molina, M.L. Brandão, Fear state induced by ethanol withdrawal may be due to the sensitization of the neural substrates of aversion in the dPAG, Exp. Neurol. 200 (2006) 200-208. https://doi.org/10.1016/j.expneurol.2006.02.004.

[29] V.M. Castilho, K.G. Borelli, M.L. Brandão, M.J. Nobre, Anxiety-like symptoms induced by morphine withdrawal may be due to the sensitization of the dorsal periaqueductal grey, Physiol. Behav. 94 (2008) 552-562. https://doi.org/10.1016/j.physbeh.2008.03.014. 


\section{Figure captions}

Figure 1 - Aminoguanidine (AG), a NOS-2 inhibitor, blocks the increases in bottom-dwelling after ethanol withdrawal in zebrafish. (A) Time spent in the bottom of the tank. (A') Detail of Figure 1A. (B) Time spent in the top of the tank. (B') Detail from Figure 1B. Data are presented as boxplots with Tukey hinges, overlapped with individual data points. Different letters indicate statistically significant differences.
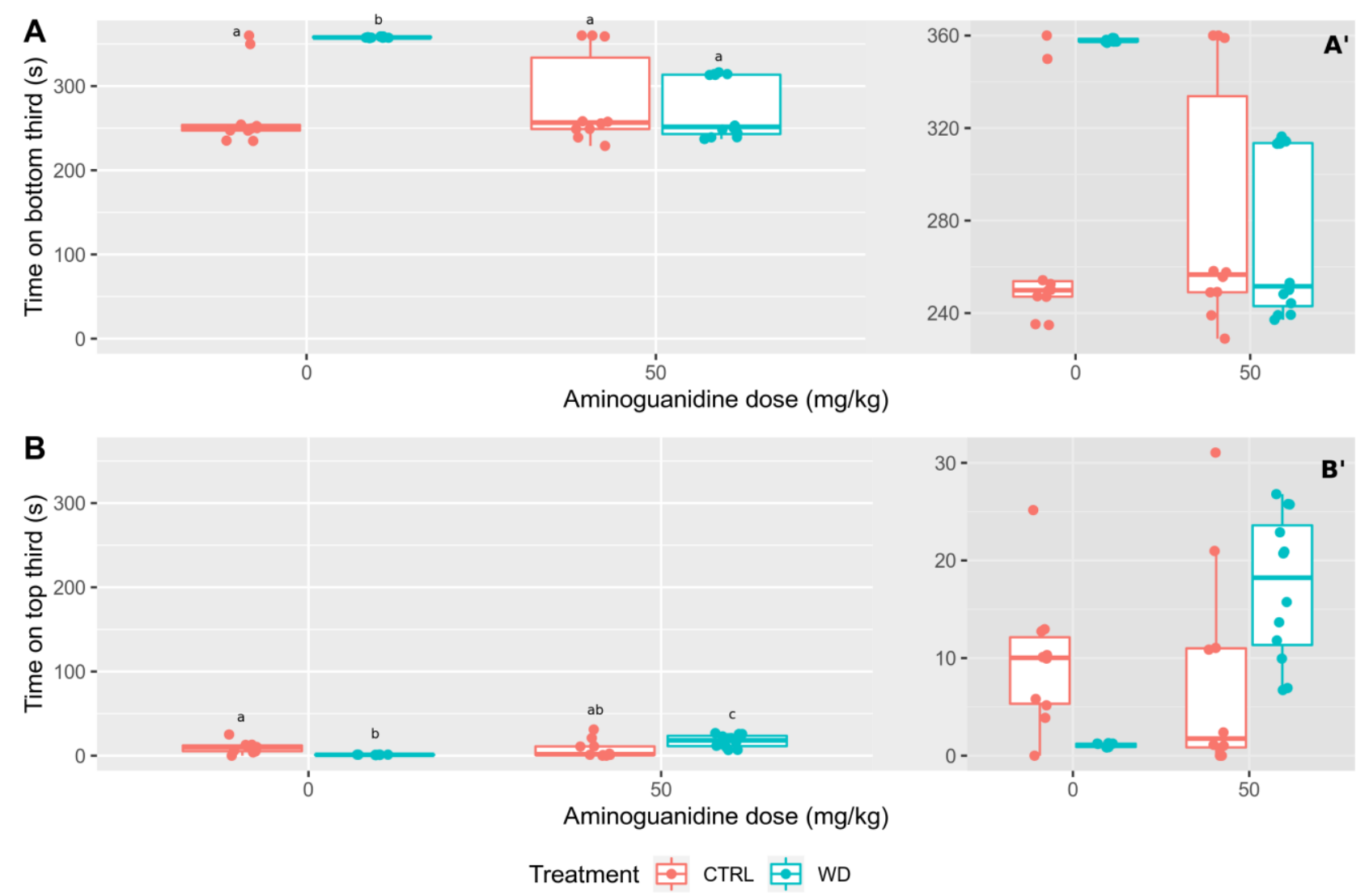

Figure 2 - Aminoguanidine (AG), a NOS-2 inhibitor, blocks the increase in freezing duration and brain nitrite levels after ethanol withdrawal in zebrafish. (A) Erratic swimming. (B) Thrashing. (C) Freezing frequency. (D) Freezing duration. (E) Number of squares crossed. (F) Brain nitrite levels. Data are presented as boxplots with Tukey hinges, overlapped with individual data points. Different letters indicate statistically significant differences. 

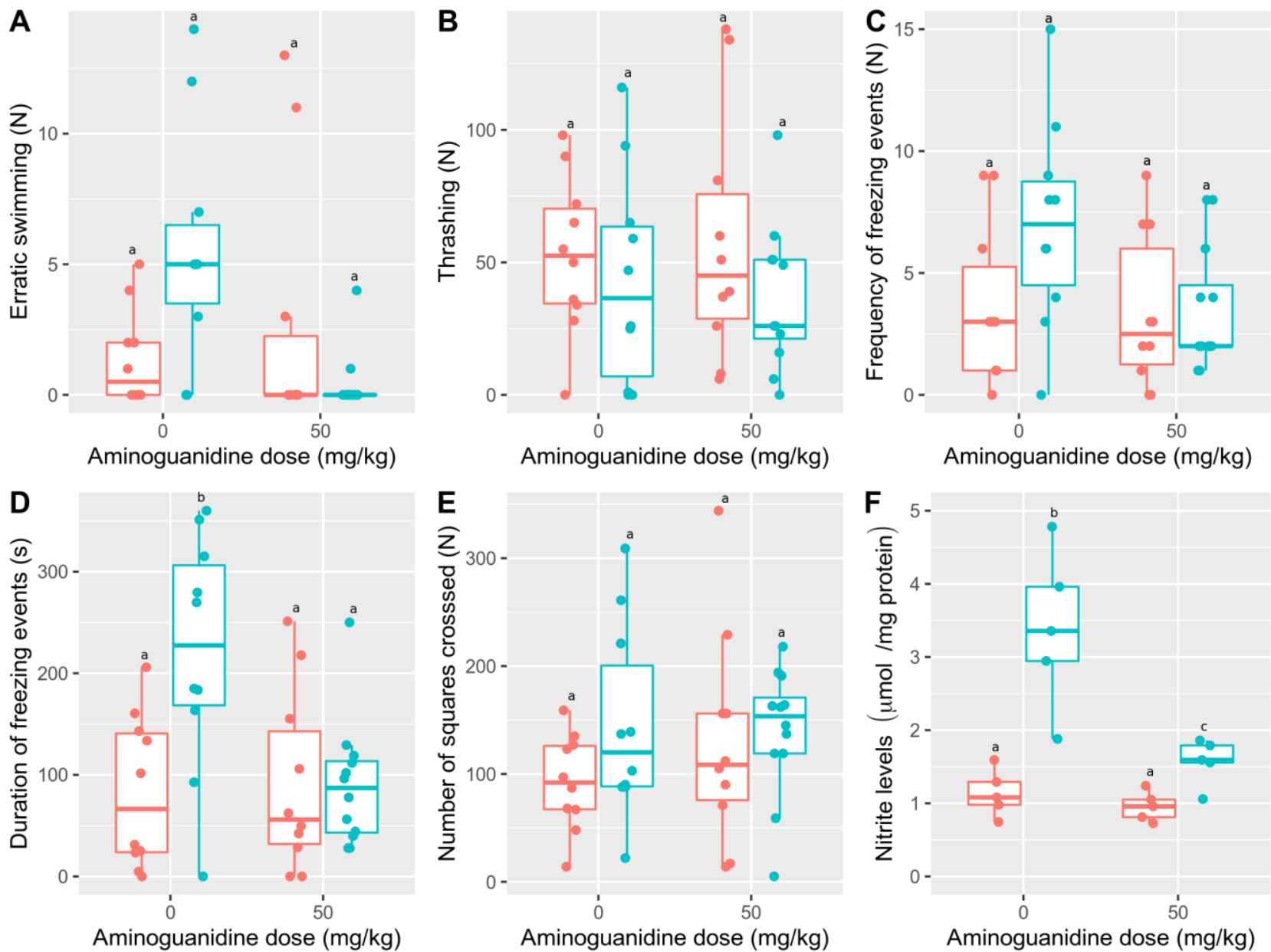

Treatment 